\title{
Coordinated transportation of a large object by a team of three robots
}

\author{
Rui Soares ${ }^{1}$ and Estela Bicho ${ }^{2}$ \\ 1 Esc. Sup. Tec. e Gestão de Felgueiras - Instituto Politecnico do Porto, Portugal \\ rsoares@estgf.ipp.pt \\ $2 \star \star$ Dep. Electrónica Industrial, Universidade do Minho, Portugal \\ estela.bicho@dei.uminho.pt
}

\begin{abstract}
Dynamical systems theory in this work is used as a theoretical language and tool to design a distributed control architecture for a team of three robots that must transport a large object and simultaneously avoid collisions with either static or dynamic obstacles. The robots have no prior knowledge of the environment. The dynamics of behavior is defined over a state space of behavior variables, heading direction and path velocity. Task constraints are modeled as attractors (i.e. asymptotic stable states) of the behavioral dynamics. For each robot, these attractors are combined into a vector field that governs the behavior. By design the parameters are tuned so that the behavioral variables are always very close to the corresponding attractors. Thus the behavior of each robot is controlled by a time series of asymptotical stable states. Computer simulations support the validity of the dynamical model architecture.
\end{abstract}

\section{INTRODUCTION}

The challenge to develop teams of autonomous robots that are able to transport large objects is an important endeavor since such multi-robot teams would be potentially useful in many fields related to our daily activities (e.g. $[1,2,4,9,10])$.

From the point of view of a robot, the environment, which comprises the others robots and the world scenario, exhibits complex dynamic behavior. The problem is exacerbated when the environment is not known and no path is given. If motion coordination of two robots carrying an object is not a trivial task, motion coordination of larger teams is undoubtedly more difficult.

Here we present results on the problem of coordinating and controlling three mobile robots that must carry a rigid object from an initial position to a final target destination.

This paper extends previous work reported in $[6,8]$. In those works dynamical systems theory was used as the theoretical framework to design and implement a distributed control architecture for a team of two mobile robots that cooperatively had to transport a long object in a cluttered environments.

Here it is assumed that: $(a)$ the robots have no prior knowledge of the environment and no path is given; $(b)$ a leader-helper decentralized motion control strategy is used, where the leader robot moves from an initial position to a final target destination (see

\footnotetext{
** corresponding author
} 
e.g. [9]); (c) each helper robot $\left(H_{1}\right.$ and $\left.H_{2}\right)$ takes the leader and the other helper robot as reference points and must maintain at all times a correct distance and orientation with them that permits it to help them in the transportation task (see Figure 1).

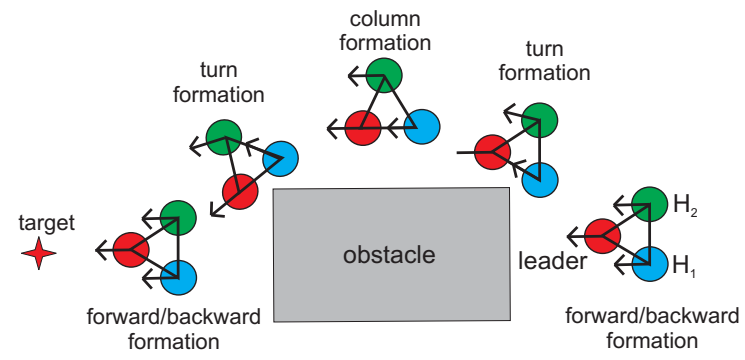

Fig. 1. Coordinated object transportation by three robots in an unknown environment. By default the robots must transport the object keeping a forward/backward formation ( $\mathrm{f} / \mathrm{b})$. When due to encountered obstacles that is not possible the helper robots must drive in turn or in column formation.

The control architecture of each robot is structured in terms of elementary behaviors. The individual behaviors and their integration are generated by non-linear dynamical systems. For each behavior, desired values for the controlled variables are identified and made attractor solutions of the dynamical systems that generates the robots' motion.

The rest of the paper is structured as follows: the next section presents the robot team, their tasks and the basic assumptions in this work. The behavioral dynamics are then defined for the robot helpers $\left(H_{1}\right.$ and $\left.H_{2}\right)$. Results obtained from computer simulations are presented in section 4 . The paper ends with a brief discussion, conclusions and an outlook of future work.

\section{ROBOT TEAM AND TASK CONSTRAINTS}

The simulated robots are based on the physical mobile robots used in [8] but the support base for the object is new. This support base gives displacements $(\Delta x, \Delta y)$ and rotation $(\Delta w)$ of the object (see Figure 2 ). The coordination and control are based on the following ideas: $(a)$ the behavior of each robot is controlled independently; $(b)$ the leader robot knows the target position, and its task consists in moving from an initial position to a final target destination; $(c)$ the task constraints for the helper robots consist in keeping at all times a correct orientation and distance with respect to the leader and the other helper; $(d)$ by default the helpers must move in an $F / B$ formation, however if obstacles do not allow it, they must adequately move in turn or column formation to guarantee obstacle avoidance for the team; $(e)$ the leader robot broadcast it's velocity and heading direction to the helpers; $(f)$ each helper from $\Delta x, \Delta y$ and $\Delta w$ is able to compute the directions at which the leader and the other helper lie from its current position and with respect to an external reference axis, i.e. $\psi_{H_{i}, \text { leader }}$ and $\psi_{H_{i}, H_{j}}$ 


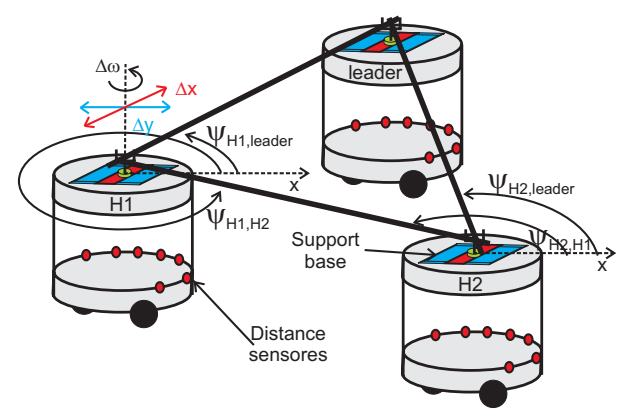

Fig. 2. Each robot has seven distance sensors mounted on a ring which is centered on the robot's rotation axis. These are used to measure the distance to obstructions at the direction in which they are pointing in space. The simulated infrared sensors have a distance range of $60 \mathrm{~cm}$ and an angular range of $30^{\circ}$. The robots are tightly coupled through a support base mounted on each robot. It consists of two prismatic and one rotational passive joints which allow to compute the directions at which each helper robot "sees" the leader and the other helper from its current position, $\psi_{H_{i}, \text { leader }}$ and $\psi_{H_{i}, H_{j}}(i=1,2 ; j=1,2 ; i \neq j)$, and displacements $(\Delta x, \Delta y)$ and orientation $(\Delta w)$ of the object.

$(i=1,2 ; j=1,2 ; i \neq j)$ respectively (see Figure 2$) ;(g)$ the helper robots broadcast their heading direction values; $(h)$ each helper broadcasts its current values of the potential function and magnitude, of the virtual obstacles avoidance dynamics; $(i)$ the helper robots do not need to know the object size, they only need to know the displacement and rotation of the transported object in their support base; $(j)$ the robots have nonholonomic motion constraints.

\section{ATTRACTOR DYNAMICS FOR COORDINATED TRANSPORTATION}

To model the robots behavior their heading direction is used, $\phi(0 \leq \phi \leq 2 \pi)$, with respect to an arbitrary but fixed world axis and path velocity, $\vartheta$. Behavior is generated by continuously providing values to these variables, which control the robot's wheels. The time course of each of these variables is obtained from (constant) solutions of dynamical systems. The attractor solutions (asymptotically stable states) dominate these solutions by design. The leader's heading direction and path velocity dynamics has been previously define, implemented and evaluated in [3], but for the obstacle avoidance dynamics the leader robot takes into account it's size as well as the dimensions of the team. $H_{i}$ 's $\left(i=H_{1}, H_{2}\right)$ heading direction and path velocity dynamics are ruled by the following dynamical systems:

$$
\begin{gathered}
\frac{d \phi_{H_{i}}(t)}{d t}=-2 \lambda_{H_{i}} \cos \left(\lambda_{\text {desired }, H_{i}}\right) \sin \left(\phi_{H_{i}}-\psi_{\text {desired }, H_{i}}\right)+f_{\text {stoch }} \\
\frac{d \vartheta_{H_{i}}(t)}{d t}=-c_{H_{i}}\left(\vartheta_{H_{i}}-V_{\text {desired }, H_{i}}\right) e^{\left[-\frac{\left(\vartheta_{H_{i}}-V_{\text {desired } H_{i}}\right)^{2}}{2 \sigma_{V}^{2}}\right]}+g_{\text {stoch }}
\end{gathered}
$$


The heading direction dynamics (1) puts an attractor at $\psi_{\text {desired, } H_{i}}$ with a strength of attraction (relaxation rate) defined by $-2 \lambda_{H_{i}} \cos \left(\lambda_{\text {desired } H_{i}}\right)$, and a repeller in the opposite direction. The path velocity dynamics (2) defines a simple attractor at the desired path velocity with a relaxation rate defined by $c_{H_{i}}$. The exponential term in (2) is used to make sure that the increasing and decreasing of velocity is smooth even when the difference $\vartheta_{H_{i}}-V_{\text {desired } H_{i}}$ is very large. $f_{\text {stoch }}$ and $g_{\text {stoch }}$ are stochastic forces that are added to the dynamical systems, to simulate perturbations and noise in the system.

In the next two subsections will be explained how the attractor values for heading direction and path velocity are computed from sensed and/or communicated information. From now on $H_{i}=H_{1}, H_{2} ; H_{j}=H_{1}, H_{2} ; H_{i} \neq H_{j}$.

\subsection{Attractor dynamics for heading direction}

The heading direction dynamics, for each helper is governed by (1) where:

$$
\begin{aligned}
& \psi_{\text {desired } H_{i}}=\gamma_{H_{i}, f / b} \psi_{\text {desired }, H_{i}, f / b}+\gamma_{H_{i}, \text { turn }} \psi_{\text {desired } H_{i}, \text { turn }}+ \\
& +\gamma_{H_{i}, \text { column }} \psi_{\text {desired }, H_{i}, \text { column }} \\
& \lambda_{\text {desired }, H_{i}}=\gamma_{H_{i}, f / b} \lambda_{\text {desired }, H_{i}, f / b}+\gamma_{H_{i}, \text { turn }} \lambda_{\text {desired } H_{i}, \text { turn }}+ \\
& +\gamma_{H_{i}, \text { column }} \lambda_{\text {desired }, H_{i}, \text { column }}
\end{aligned}
$$

Here $\psi_{\text {desired } H_{i}, f / b}, \psi_{\text {desired } H_{i}, \text { turn }}$ and $\psi_{\text {desired } H_{i}, \text { column }}$ are the desired directions at which the attractors emerge for each different behavior, forward/backward, turn and column formations, respectively (see Figure 3). $\gamma_{H_{i}, f / b}, \gamma_{H_{i}, \text { turn }}$ and $\gamma_{H_{i}, \text { column }}$ are mutually exclusive activation variables that determine which attractor value must dominate the dynamics. In the next two subsections, it will be explained how the activation variables and the attractor values are computed from sensed and/or communicated information.

\section{Activation variables}

Forward/backwardformation: In the absence of obstacles robot $H_{i}$ must move in a "f/b formation", so the term $\gamma_{H_{i}, f / b}$ must dominate the dynamics. Thus $\gamma_{H_{i}, f / b}=1$, $\gamma_{H_{i}, \text { turn }}=0$ and $\gamma_{H_{i}, \text { column }}=0$ is required.

$$
\gamma_{H_{i}, f / b}=\left\{\begin{array}{l}
+1, \text { if } \alpha_{f_{i}}=1 \vee \alpha_{b_{i}}=1 \\
0, \text { else }
\end{array}\right.
$$

where $\alpha_{f_{i}}$ and $\alpha_{b_{i}}$ signal the control architecture when it's necessary for the helper robots to move forward or backward respectively ${ }^{3}$ (see Figure 4):

$\alpha_{f_{i}}=\left\{\begin{array}{c}+1, \text { if }\left[\left(U_{o b s, H_{i}}>0 \wedge U_{o b s, H_{j}}>0\right) \vee\left(U_{o b s, H_{i}} \leq 0 \wedge U_{o b s, H_{j}} \leq 0\right)\right] \wedge \\ 0, \text { else }\left[\left|\phi_{H_{i}}-\phi_{\text {leader }}\right|<\Delta \theta_{1} \wedge\left|\phi_{H_{j}}-\phi_{\text {leader }}\right|<\Delta \theta_{1}\right]\end{array}\right.$

\footnotetext{
${ }^{3} \Delta \theta_{1}$ is a constant. Here equal to $90^{\circ}$
} 


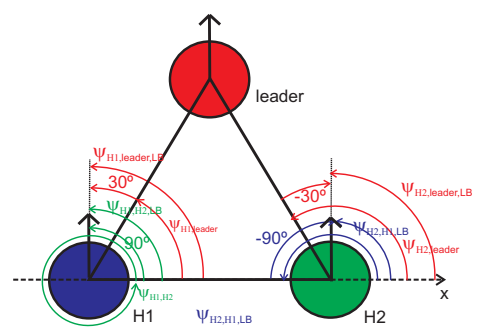

FB formation

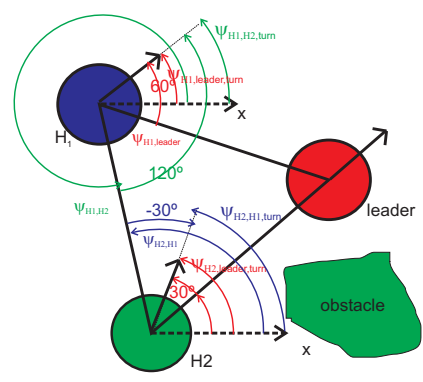

Turn formation

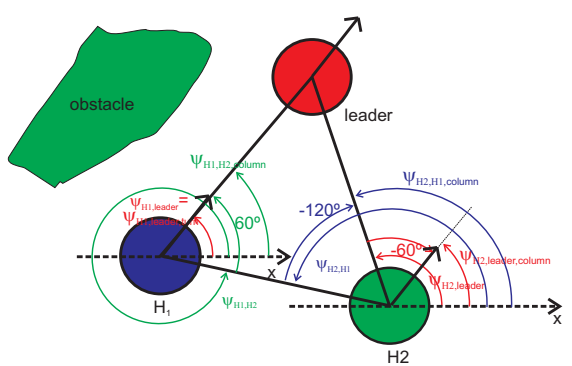

Column formation

Fig. 3. Desired directions for each helper robot for the different behaviors.

$\alpha_{b_{i}}=\left\{\begin{array}{c}+1, \text { if }\left[\left(U_{\text {obs }, H_{i}}>0 \wedge U_{o b s, H_{j}}>0\right) \vee\left(U_{\text {obs }, H_{i}} \leq 0 \wedge U_{o b s, H_{j}} \leq 0\right)\right] \wedge \\ 0, \text { else } \\ {\left[\left|\phi_{H_{i}}-\phi_{\text {leader }}\right| \geq \Delta \theta_{1} \wedge\left|\phi_{H_{j}}-\phi_{\text {leader }}\right| \geq \Delta \theta_{1}\right]}\end{array}\right.$

This permits robot $H_{i}$ to move forward or backward keeping a $f / b$ formation.
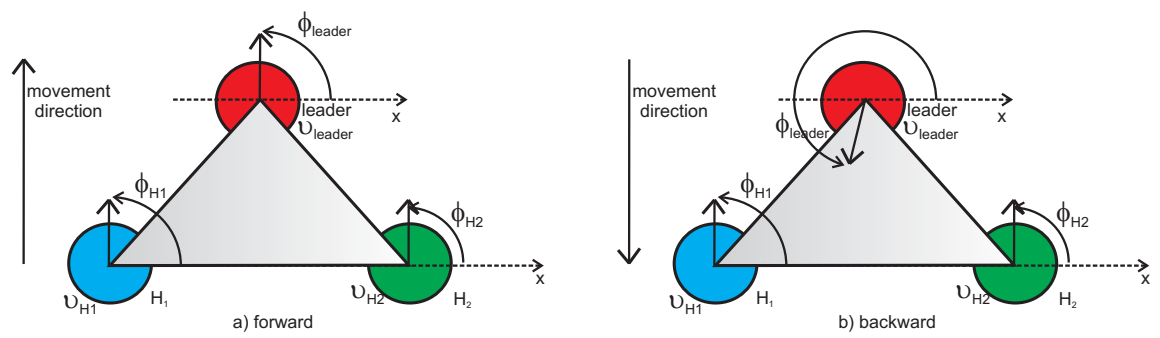

Fig. 4. (a) The heading direction of the leader robot isn't pointing to a direction between the two helper robots, the helper robots must move forward; (b) The heading direction of the leader robot is pointing to a direction between the two helper robots, the helper robots must move backward.

Turn formation: When obstructions are detected and the difference between the direc- 
tion $\psi_{H_{i}, \text { leader }}$ and $\phi_{\text {leader }}$ is larger than a certain value, $\Delta \theta_{2}{ }^{4}$, we want that the robots to move in turn formation, so the term $\gamma_{H_{i}, \text { turn }}$ must dominate the dynamics. Thus $\gamma_{H_{i}, \text { turn }}=1, \gamma_{H_{i}, f / b}=0$ and $\gamma_{H_{i}, \text { column }}=0$. This implies robot $H_{i}$ to turn around, i.e. avoid the obstacle by turning to the right or to the left. Robot $H_{i}$ takes this decision based on:

$\alpha_{t r_{i}}=\left\{\begin{array}{l}+1, \text { if } U_{\text {obs }, H_{i}}>0 \wedge U_{\text {obs }, H_{j}} \leq 0 \wedge F_{\text {obs }, H_{i}}<0 \wedge\left|\phi_{\text {leader }}-\psi_{H_{i}, \text { leader }}\right|>\Delta \theta_{2} \\ 0, \text { else }\end{array}\right.$

$\alpha_{t l_{i}}=\left\{\begin{array}{l}+1, \text { if } U_{o b s, H_{i}}>0 \wedge U_{o b s, H_{j}} \leq 0 \wedge F_{\text {obs }, H_{i}}>0 \wedge\left|\phi_{\text {leader }}-\psi_{H_{i}, \text { leader }}\right|>\Delta \theta_{2} \\ 0, \text { else }\end{array}\right.$

where $\alpha_{t r_{i}}$ and $\alpha_{t l_{i}}$ signal the control architecture when it's necessary robot $H_{i}$ to turn right or left respectively. $U_{o b s, H_{i}}$ and $U_{o b s, H_{j}}$ in (8) and (9) are the potential functions of the virtual obstacles avoidance dynamics for each helper robot, that indicate if obstacles contributions are present (how to compute these see $[6,8]$ ). Positive values of these functions indicate that the helpers heading direction are in a repulsion zone of sufficient strength. Conversely, negative values of these functions indicates that the heading direction is outside the repulsion range or the repulsion is very weak. As shown in [6] and [8] the virtual obstacle avoidance dynamics, $F_{o b s, H_{i}}$ and $F_{o b s, H_{j}}$, can be used to signal if obstacles are to the right or left side of robots. Positive values of these functions indicate that an obstacle is detected at the right side of the robot. Conversely, negative values of these functions indicate that an obstacle is detected on the left side of the robots. Finally activation variable $\gamma_{H_{i}, \text { turn }}$ is set by:

$$
\gamma_{H_{i}, \text { turn }}=\left\{\begin{array}{l}
+1, \text { if } \alpha_{t r_{i}}=1 \vee \alpha_{t l_{i}}=1 \\
0, \text { else }
\end{array}\right.
$$

Column formation: If obstructions are detected and the difference between the direction $\bar{\psi}_{H_{i}, \text { leader }}$ and $\phi_{\text {leader }}$ is smaller than $\Delta \theta_{2}$, the term $\gamma_{H_{i}, \text { column }}$ must dominate the dynamics, so $\gamma_{H_{i}, \text { column }}=1, \gamma_{H_{i}, f / b}=0$ and $\gamma_{H_{i}, \text { turn }}=0$. This makes robot $H_{i}$ to move in parallel with the detected obstacle, i.e. to move in right or left column. Robot $H_{i}$ takes this decision as defined by:

$$
\begin{aligned}
\alpha_{c r_{i}} & =\left\{\begin{array}{l}
+1, \text { if } U_{o b s, H_{i}} \leq 0 \wedge U_{o b s, H_{j}}>0 \wedge\left|\phi_{\text {leader }}-\psi_{H_{i}, \text { leader }}\right| \leq \Delta \theta_{2} \\
0, \text { else }
\end{array}\right. \\
\alpha_{c l_{i}} & =\left\{\begin{array}{l}
+1, \text { if } U_{o b s, H_{i}}>0 \wedge U_{o b s, H_{j}} \leq 0 \wedge\left|\phi_{\text {leader }}-\psi_{H_{i}, \text { leader }}\right| \leq \Delta \theta_{2} \\
0, \text { else }
\end{array}\right.
\end{aligned}
$$

where $\alpha_{c r_{i}}$ and $\alpha_{c l_{i}}$ signal right or left column respectively. $\gamma_{H_{i}, \text { column }}$ can then be set:

$$
\gamma_{H_{i}, \text { column }}=\left\{\begin{array}{l}
+1, \text { if } \alpha_{c r_{i}}=1 \vee \alpha_{c l_{i}}=1 \\
0, \text { else }
\end{array}\right.
$$

\footnotetext{
${ }^{4} \Delta \theta_{2}$ is a constant. Here equal to $5^{\circ}$. This value is the minimal value obtained by experimental simulations for a safe turn formation for each helper robot without losing the object.
} 
Attractor values for different behaviors From (3) it is possible to see one desired direction for each different behavior (i.e. $\psi_{\text {desired }{ }_{H}, f / b}, \psi_{\text {desired }, H_{i}, \text { turn }}$ and $\psi_{\text {desired }, H_{i}, \text { column }}$ ) (see Figure 3).

Forward/backward formation: The desired attractor and its strengths for $F / B$ formation are given by:

$$
\begin{gathered}
\psi_{H_{i}, f / b}=\frac{\psi_{H_{i}, \text { leader }, f / b}+\psi_{H_{i}, H_{j}, f / b}}{2} \\
\lambda_{H_{i}, f / b}=\frac{-\psi_{H_{i}, l e a d e r, f / b}+\psi_{H_{i}, H_{j}, f / b}}{2}
\end{gathered}
$$

where $\psi_{H_{i}, \text { leader, } f / b}$ is the desired attractor for $H_{i}$ robot with respect to the leader robot (see Figure 5(a)) and $\psi_{H_{i}, H_{j}, f / b}$ is the desired attractor for $H_{i}$ robot with respect to $H_{j}$ robot (see Figure 5(b)).

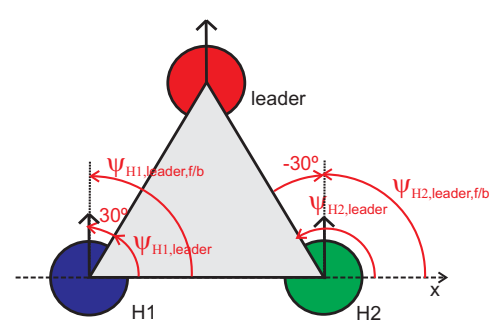

a)

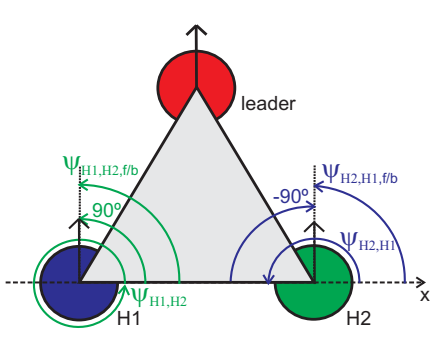

b)

Fig. 5. (a) Desired direction for each helper robot with respect to the leader robot; (b) Desired direction for each helper robot with respect to the other. (parameters settings: $k_{H_{1}}=1, k_{H_{2}}=$ $-1, \delta_{f / b, H_{i}, \text { leader }}=30^{\circ}, \delta_{f / b, H_{i}, H_{j}}=90^{\circ}, R_{H_{1}}=1, R_{H_{2}}=-1, \Delta_{H_{i}}=0$.)

Desired direction of $H_{i}$ with respect to the leader robot: As depicted in Figure 5(a), the attractor is set at a direction

$$
\psi_{H_{i}, \text { leader }, f / b}=\psi_{H_{i}, \text { leader }}+k_{H_{i}} \delta_{f / b, H_{i}, \text { leader }}+R_{H_{i}} \Delta_{H_{i}}
$$

where $\psi_{H_{i}, \text { leader }}$ is the direction at which $H_{i}$ robot "sees" the leader from its current position. $k_{H_{i}}$ is a parameter that can take the values -1 or +1 depending on the robot that is referred to.

$$
k_{H_{i}}=\left\{\begin{array}{l}
+1 \text { if } H_{i}=H_{1} \\
-1 \text { if } H_{i}=H_{2}
\end{array}\right.
$$

$\delta_{f / b, H_{i}, \text { leader }}$ is a constant (here equal to $30^{\circ}$ ). $R_{H_{i}}$ is a constant that can take one of two values, -1 or +1 , depending on the helper robot that is referred to and at the parameter $\alpha_{b_{i}}$ (7),

$$
R_{H_{i}}= \begin{cases}+1 \text { if }\left(H_{i}=H_{1} \wedge \alpha_{b_{i}}=0\right) & \vee\left(H_{i}=H_{2} \wedge \alpha_{b_{i}}=1\right) \\ -1 \text { if }\left(H_{i}=H_{1} \wedge \alpha_{b_{i}}=1\right) & \vee\left(H_{i}=H_{2} \wedge \alpha_{b_{i}}=0\right) .\end{cases}
$$


$\Delta_{H_{i}}$ in (16), is a sigmoidal function that varies with the displacements of the object measured by the support base, i.e. it converts the displacement of the object into an angle that approaches null as the displacements of the object tends to zero and it is given by:

$$
\Delta_{H_{i}}=\frac{2 \arctan \left(\alpha_{H_{i}} \Delta d_{H_{i}}\right)}{\pi}
$$

where $\alpha_{H_{i}}{ }^{5}$ is a constant and $\Delta d_{H_{i}}$ is the displacement of the object measured by the support base of each helper robot.

Desired direction of $H_{i}$ with respect to the $H_{j}$ robot: Figure 5(b), shows that the desired attractor is set at a direction

$$
\psi_{H_{i}, H_{j}, f / b}=\psi_{H_{i}, H_{j}}+k_{H_{i}} \delta_{f / b, H_{i}, H_{j}}+R_{H_{i}} \Delta_{H_{i}}
$$

where $k_{H_{i}}, R_{H_{i}}$ and $\Delta_{H_{i}}$ are the same parameters used in (17), (18) and (19), respectively. $\psi_{H_{i}, H_{j}}$ is the direction at which helper $H_{i}$ "sees" helper $H_{j}$ from it's current position. $\delta_{f / b, H_{i}, H_{j}}$ its an angle (here equal to $90^{\circ}$ ).

Turn formation: When moving around an obstacle the desired attractor and strength for turn formation are given by:

$$
\begin{gathered}
\psi_{H_{i}, \text { turn }}=\frac{\psi_{H_{i}, \text { leader }, \text { turn } n}+\psi_{H_{i}, H_{j}, \text { turn }}}{2} \\
\lambda_{H_{i}, \text { turn }}=\frac{-\psi_{H_{i}, \text { leader }, \text { turn }}+\psi_{H_{i}, H_{j}, \text { turn }}}{2}
\end{gathered}
$$

where $\psi_{H_{i}, \text { leader,turn }}$ is the desired attractor of $H_{i}$ robot with respect to the leader robot (see Figure 6(a)) and $\psi_{H_{i}, H_{j}, \text { turn }}$ is the desired attractor of $H_{i}$ robot with respect to $H_{j}$ robot (see Figure 6(b)).

Desired direction of $H_{i}$ with respect to the leader robot: As it is possible to see in Figure 6(a), the desired attractor emerges at a direction

$$
\psi_{H_{i}, \text { leader,turn }}=\psi_{H_{i}, \text { leader }}+\delta_{\text {turn }, H_{i}, \text { leader }}+R_{H_{i}} \Delta_{H_{i}}
$$

where $\Delta_{H_{i}}$ is given by (19). $R_{H_{i}}$ is a parameter that takes the value 1 or -1 depending on the helper robot are we referring to.

$$
R_{H_{i}}= \begin{cases}+1 & \text { if }\left(H_{i}=H_{1}\right) \\ -1 & \text { if }\left(H_{i}=H_{2}\right)\end{cases}
$$

$\psi_{H_{i}, \text { leader }}$ is the direction at which $H_{i}$ robot "sees" the leader. $\delta_{\text {turn, } H_{i}, \text { leader }}$ is an angle that depends in what direction helper $H_{i}$ has to turn to, left or right.

$$
\delta_{\text {turn }, H_{i}, \text { leader }}=\left\{\begin{array}{l}
\Delta \psi_{H_{i}, \text { leader }, \text { turn left }} \text { if } \alpha_{t l_{i}}=1 \\
-\Delta \psi_{H_{i}, \text { leader }, \text { turn right }} \text { else }
\end{array}\right.
$$

\footnotetext{
${ }^{5}$ Here, $\alpha_{H_{1}}=0.1, \alpha_{H_{2}}=1$
} 


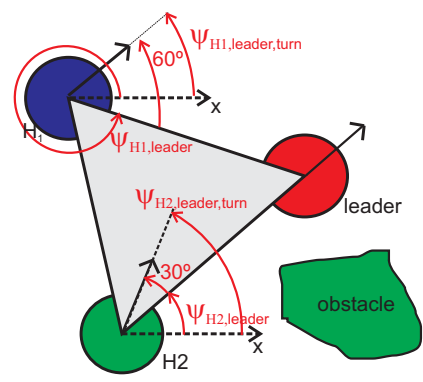

a)

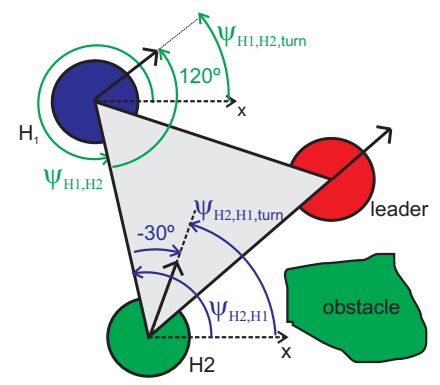

b)

Fig. 6. (a)Desired direction for each helper robot with respect to the leader robot; (b) Desired direction for each helper robot with respect to the other. (parameters settings: $\delta_{\text {turn, } H_{1}, \text { leader }}=$ $60^{\circ}, \delta_{\text {turn }, H_{2}, \text { leader }}=30^{\circ}, \delta_{\text {turn }, H_{1}, H_{2}}=120^{\circ}, \delta_{\text {turn }, H_{2}, H_{1}}=30^{\circ}, R_{H_{1}}=1, R_{H_{2}}=-1$, $\left.\Delta_{H_{i}}=0.\right)$

where $\alpha_{t l_{i}}$ is given by (9).

$\Delta \psi_{H_{i}, \text { leader,turn left }}$ and $\Delta \psi_{H_{i}, \text { leader,turn right }}$ are angles that depend on what helper robot is referred to. Here

$$
\begin{gathered}
\Delta \psi_{H_{i}, \text { leader,turn left }}=\left\{\begin{array}{l}
60^{\circ} \text { if } H_{i}=H_{1} \\
30^{\circ} \text { if } H_{i}=H_{2}
\end{array}\right. \\
\Delta \psi_{H_{i}, \text { leader,turn right }}=\left\{\begin{array}{l}
30^{\circ} \text { if } H_{i}=H_{1} \\
60^{\circ} \text { if } H_{i}=H_{2}
\end{array}\right.
\end{gathered}
$$

Desired direction of $H_{i}$ with respect to the $H_{j}$ robot: In Figure 6(b), it's possible to see that the desired attractor is built at a direction

$$
\psi_{H_{i}, H_{j}, t u r n}=\psi_{H_{i}, H_{j}}+\delta_{\text {turn }, H_{i}, H_{j}}+R_{H_{i}} \Delta_{H_{i}}
$$

where $R_{H_{i}}$ and $\Delta_{H_{i}}$ are given by (24) and (19), respectively. $\psi_{H_{i}, H_{j}}$ is the direction at which $H_{i}$ robot sees $H_{j}$ robot. $\delta_{t u r n, H_{i}, H_{j}}$ it's an angle that depends in what direction the helper $H_{i}$ has to turn to, left or right.

$$
\delta_{\text {turn }, H_{i}, H_{j}}=\left\{\begin{array}{l}
R_{H_{i}} \Delta \psi_{H_{i}, H_{j}, \text { turn left }} \text { if } \alpha_{t l_{i}}=1 \\
R_{H_{i}} \Delta \psi_{H_{i}, H_{j}, \text { turn right }} \text { else }
\end{array}\right.
$$

where $\alpha_{t l_{i}}$ is given by (9). $\Delta \psi_{H_{i}, H_{j}, \text { turn left }}$ and $\Delta \psi_{H_{i}, H_{j}, t u r n ~ r i g h t}$ are angles that depend on what robot helper we are referring to. Here

$$
\begin{gathered}
\Delta \psi_{H_{i}, H_{j}, \text { turn left }}=\left\{\begin{array}{l}
120^{\circ} \text { if } H_{i}=H_{1} \\
30^{\circ} \text { if } H_{i}=H_{2}
\end{array}\right. \\
\Delta \psi_{H_{i}, H_{j}, \text { turn right }}=\left\{\begin{array}{l}
30^{\circ} \text { if } H_{i}=H_{1} \\
120^{\circ} \text { if } H_{i}=H_{2}
\end{array}\right.
\end{gathered}
$$


Column formation: The desired attractor and strength for column formation are given by:

$$
\begin{gathered}
\psi_{H_{i}, \text { column }}=\frac{\psi_{H_{i}, \text { leader }, \text { column }}+\psi_{H_{i}, H_{j}, \text { column }}}{2} \\
\lambda_{H_{i}, \text { column }}=\frac{-\psi_{H_{i}, \text { leader }, \text { column }}+\psi_{H_{i}, H_{j}, \text { column }}}{2}
\end{gathered}
$$

where $\psi_{H_{i}, \text { leader,column }}$ is the desired attractor of $H_{i}$ robot with respect to the leader robot (see Figure $7(\mathrm{a})$ ) and $\psi_{H_{i}, H_{j}, \text { column }}$ is the desired attractor of $H_{i}$ robots with respect to each other (see Figure 7(b)).

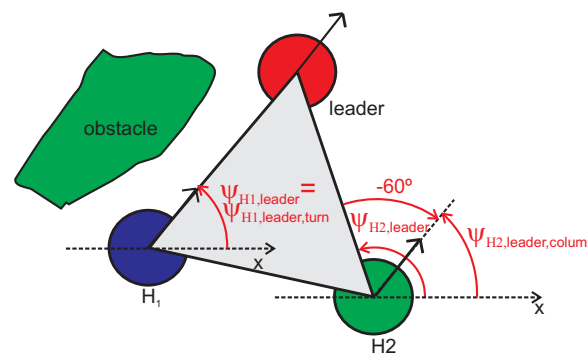

a)

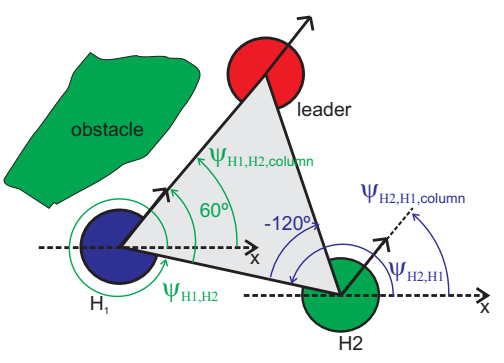

b)

Fig. 7. (a)Desired direction for each helper robot with respect to the leader robot; (b) Desired direction for each helper robot with respect to the other. (parameters settings: $\delta_{\text {column, } H_{1}, \text { leader }}=$ $0^{\circ}, \delta_{\text {column }, H_{2}, \text { leader }}=60^{\circ}, \delta_{\text {column }, H_{1}, H_{2}}=60^{\circ}, \delta_{\text {column }, H_{2}, H_{1}}=120^{\circ}, R_{H_{1}}=1$, $R_{H_{2}}=-1, \Delta_{H_{i}}=0$.)

Desired direction of $H_{i}$ with respect to the leader robot: As it is possible to see in Figure 7(a), the desired attractor is built at a direction

$$
\psi_{H_{i}, \text { leader }, \text { column }}=\psi_{H_{i}, \text { leader }}+\delta_{\text {column }, H_{i}, \text { leader }}+R_{H_{i}} \Delta_{H_{i}}
$$

where $R_{H_{i}}$ and $\Delta_{H_{i}}$ are given by (24) and (19), respectively. $\psi_{H_{i}, \text { leader }}$ is the direction at which $H_{i}$ robot "sees" the leader robot. $\delta_{\text {column, } H_{i}, \text { leader }}$ is an angle that depends on which helper robot has to be behind the leader robot, i.e. move in column, left or right.

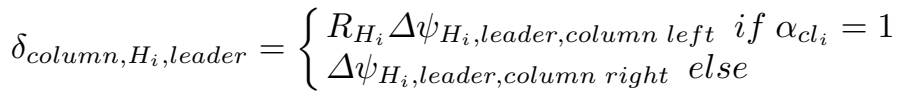

where $R_{H_{i}}$ and $\alpha_{c l_{i}}$ are given by (24) and (12), respectively.

$\Delta \psi_{H_{i}, \text { leader,column left }}$ and $\Delta \psi_{H_{i}, \text { leader,column right }}$ are angles that depend on what robot we are referring to. Here

$$
\begin{gathered}
\Delta \psi_{H_{i}, \text { leader, column left }}=\left\{\begin{array}{l}
0^{\circ} \text { if } H_{i}=H_{1} \\
60^{\circ} \text { if } H_{i}=H_{2}
\end{array}\right. \\
\Delta \psi_{H_{i}, \text { leader,column right }}=\left\{\begin{array}{l}
60^{\circ} \text { if } H_{i}=H_{1} \\
0^{\circ} \text { if } H_{i}=H_{2}
\end{array}\right.
\end{gathered}
$$


Desired direction of $H_{i}$ with respect to the $H_{j}$ robot: In Figure 7(b), it is possible to see that the desired attractor emerges at a direction

$$
\psi_{H_{i}, H_{j}, \text { column }}=\psi_{H_{i}, H_{j}}+\delta_{\text {column } H_{i}, H_{j}}+R_{H_{i}} \Delta_{H_{i}}
$$

where $R_{H_{i}}$ and $\Delta_{H_{i}}$ are given by (24) and (19), respectively. $\psi_{H_{i}, H_{j}}$ is the direction at which $H_{i}$ robot "sees" $H_{j}$ robot. $\delta_{\text {turn }, H_{i}, H_{j}}$ is an angle that depends on which helper has to be behind the leader robot.

$$
\delta_{\text {column }, H_{i}, H_{j}}=\left\{\begin{array}{l}
R_{H_{i}} \Delta \psi_{H_{i}, H_{j}, \text { column left }} \text { if } \alpha_{\text {cl }_{i}}=1 \\
R_{H_{i}} \Delta \psi_{H_{i}, H_{j}, \text { column right }} \text { else }
\end{array}\right.
$$

where $\alpha_{c l_{i}}$ is given by (12). $\Delta \psi_{H_{i}, H_{j}, \text { column left }}$ and $\Delta \psi_{H_{i}, H_{j}, \text { column right }}$ are angles that depend on what helper robot we are referring to. Here

$$
\begin{gathered}
\Delta \psi_{H_{i}, H_{j}, \text { column left }}=\left\{\begin{array}{l}
60^{\circ} \text { if } H_{i}=H_{1} \\
120^{\circ} \text { if } H_{i}=H_{2}
\end{array}\right. \\
\Delta \psi_{H_{i}, H_{j}, \text { column right }}=\left\{\begin{array}{l}
120^{\circ} \text { if } H_{i}=H_{1} \\
60^{\circ} \text { if } H_{i}=H_{2} .
\end{array}\right.
\end{gathered}
$$

\subsection{Attractor dynamics for velocity}

For the leader's path velocity control see [6]. The helpers' path velocity must be controlled so that at all times each helper robot attempts to maintain a null displacement of the object (i.e $\Delta d_{H_{i}}=0$ ). The leader robot communicates its current path velocity to the helpers. The attractor value, i.e. the required velocity $V_{\text {desired, } H_{i}}$, for the velocity dynamics (2) is given by:

$$
V_{\text {desired }, H_{i}}= \begin{cases}+\vartheta_{\text {leader }}+\frac{\left|\Delta d_{H_{i}}\right|}{\nu_{H_{i}}}, & \text { if } \Delta d_{H_{i}}<0 \\ -\vartheta_{\text {leader }}-\frac{\left|\Delta d_{H_{i}}\right|}{\nu_{H_{i}}}, & \text { else }\end{cases}
$$

$\Delta d_{H_{i}}$ is the displacement of the object measured in the support base mounted on the $H_{i}$ robot. $\nu_{H_{i}}$ is a parameter that depends on the helper robot and on what side the helper has to turn to.

$$
\nu_{H_{i}}=\left\{\begin{array}{l}
\nu_{1}, \text { if }\left(H_{i}=H_{1} \wedge \alpha_{t l_{i}}=1\right) \vee\left(H_{i}=H_{2} \wedge \alpha_{t l_{i}}=0\right) \\
\nu_{2} \text { else }
\end{array}\right.
$$

$\alpha_{t l_{i}}$ is defined by (9). $\nu_{1}{ }^{6}$ and $\nu_{2}{ }^{7}$ values were reached by simulation experiments, these values are the minimal values obtained for a safe transportation.

\subsection{Hierarchy of relaxation rates}

The following hierarchy of relaxation rates ensures that the heading direction of each helper relaxes to a dominant attractor solution.

$$
\begin{gathered}
c_{H_{i}}>>2 \lambda_{H_{i}} \cos \left(\lambda_{\text {desired }, H_{i}, f / b}\right), c_{H_{i}}>>2 \lambda_{H_{i}} \cos \left(\lambda_{\text {desired }, H_{i}, \text { turn }}\right), \\
c_{H_{i}}>>2 \lambda_{H_{i}} \cos \left(\lambda_{\text {desired }, H_{i}, \text { column }}\right)
\end{gathered}
$$

\footnotetext{
${ }^{6} \nu_{1}$ is a constant. Here equal to 3 .

${ }^{7} \nu_{2}$ is a constant. Here equal to 9 .
} 


\section{RESULTS}

The complete distributed dynamical architecture was evaluated in computer simulations, generated by software written in MATLAB. In the simulation, the robots are represented by two Cartesian coordinates and the heading direction. Cartesian coordinates are updated by a dead-reckoning rule, while the heading direction and path velocity are obtained from the corresponding behavioral dynamical. All dynamic equations are integrated with a forward Euler method with a fixed time step, and sensory information is computed once per each cycle. Distance sensors are simulated through an algorithm reminiscent of ray tracing. The target information is defined by a goal position in space. It's assumed that the leader robot communicates to the helpers its current path velocity and heading velocity. The helper robots communicate each other their current heading directions and its current values of the potential function and magnitude, of the virtual obstacles avoidance dynamics.

A simulation run in an environment with obstacles (static and dynamic), that demonstrates some features of the distributed dynamic control architecture, is presented in Figure 8 . The dynamic obstacles are represented by two robots, $R 3$ and $R 4$. Their behavioral dynamics is reported in [7]. Figure 9 shows the heading direction dynamics for each robot, at the points shown in snapshot $\mathrm{D}$. We only present one simulation run and the heading direction at one snapshot due to limitations of space (more videos can be found in www.dei.uminho.pt/pessoas/estela/).

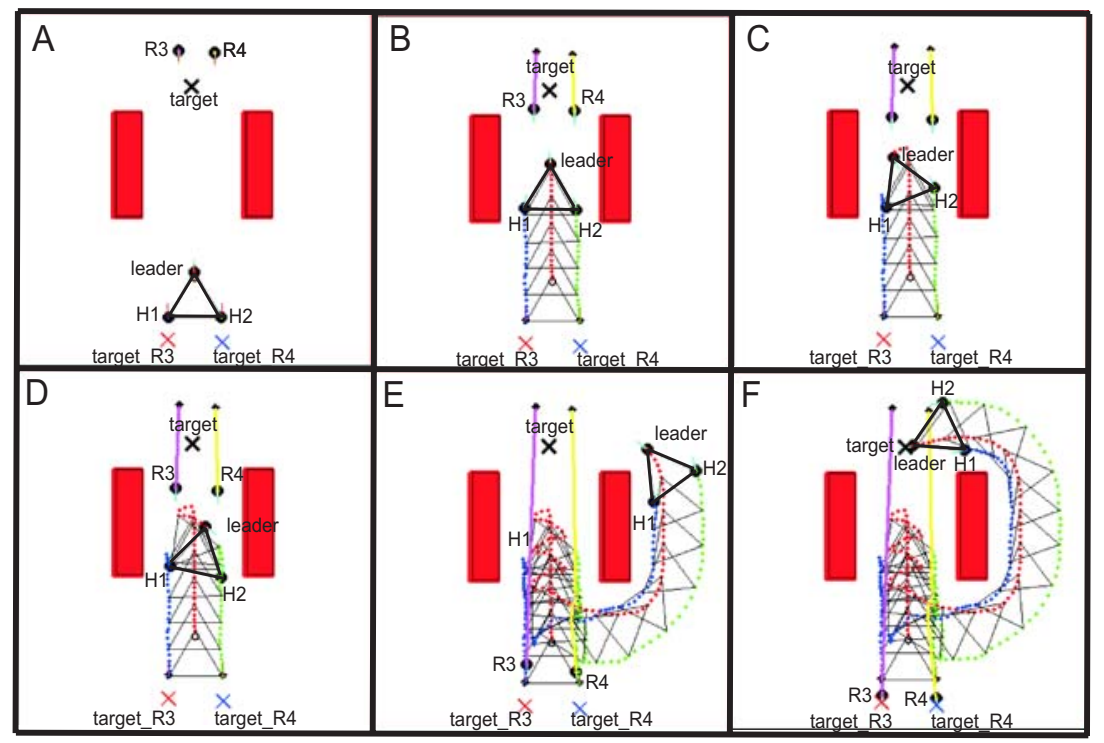

Fig. 8. Snapshots of a simulation run the complete system. Time evolves from A to F.

In Figure 8 the targets are represented by a cross. The targets of leader, $R 3$ and $R 4$ are target, target $\_3$ and target $R 4$, respectively. Each robot is represented by a 
black circle with a line that represents its heading direction. Initially the robots are placed as illustrated in Panel A. The leader moves toward its target entering in a passage between two obstacles (Panel B), and $H_{1}$ and $H_{2}$ robots start steering forward to keep a $f / b$ forward formation with the leader robot. Robots $R 3$ and $R 4$ enter in the same passage to reach their targets, i.e. target $R 3$ and target $R 4$, respectively. Then the leader robot detects these robots (Panel $\mathrm{C}$ ). This forces the leader to turn back and to change its course to avoid collisions with R3 and R4. $H_{1}$ and $H_{2}$ maintain a f/b backward formation with the leader robot while driving backwards in the passage. The other two robots continue moving in the direction of their targets. The leader robot continues trying to leave the passage (Panel D) and the helpers maintain a f/b backward formation. The robots team leaves then the passage and find a new way in the target direction (Panel E). Robots $H_{1}$ and $H_{2}$ maintain a turn formation with the leader. Finally all robots reach their targets (Panel F).

The heading directions dynamics for the three robots in the team can be seen in Figure 9 at the position depicted at snapshot D. The black arrow in each plot indicates the current state (i.e. heading direction in the world) of the corresponding robot. As it's possible to see, the heading direction of each robot is always very close to a fixed point attractor (i.e. in the Figure 9 is the one with negative slope) of the resultant dynamics.

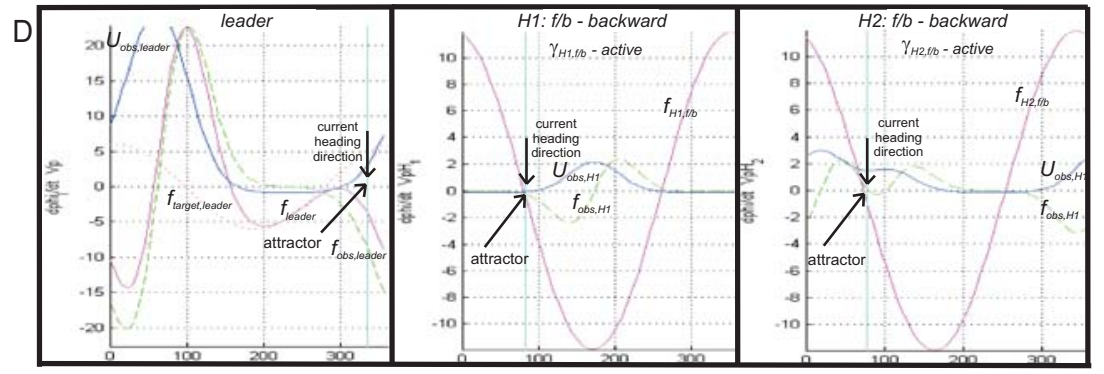

Fig. 9. Heading direction dynamics for the three robots in the team when they are at positions depicted in Figure 8. Left plot: it's possible to see contributions of sensed obstacles $\left(f_{\text {obs }}\right.$, leader $)$ and target contribution $\left(f_{\text {target,leader }}\right)$. The resultant dynamics is $f_{\text {leader }}$. Middle plot: here the robot senses obstructions. Its heading direction is inside the repulsive range and the difference between the $H_{1}$ robot heading direction and the heading direction of the leader robot is greater than $90^{\circ}$, and also the difference between the $H_{2}$ robot heading direction and the heading direction of the leader robot is greater than $90^{\circ}$. Thus the resultant dynamics is dominated by the term $\gamma_{H_{1}, f / b}$. Right plot: this robot also senses obstructions. Its heading direction is inside the repulsive range and the difference between the $H_{2}$ robot heading direction and the heading direction of the leader robot is greater than $90^{\circ}$, and also the difference between the $H_{1}$ robot heading direction and the heading direction of the leader robot is greater than $90^{\circ}$. Thus the resultant dynamics is dominated by the term $\gamma_{H_{2}, f / b}$. 


\section{CONCLUSIONS AND FUTURE WORK}

In this paper, non-linear attractor dynamics was used as a tool to design a distributed control architecture that enables a team of three robots to transport a large object. It was assumed that the robots have no prior knowledge of the environment. The choice of the control variables and parameters have taken into account the physical mobile robots at which the architecture will be implemented. The amount of information communicated among robots is minimal. The overall control system is flexible, since planning solutions may change based on changes in sensed world and/or communicated information. The leader broadcast its heading direction and path velocity (codified in 2 bytes) and each helper share among them 3 values represented by 3 bytes. The control architecture was evaluated through computer simulations. The global behavior is stable and trajectories are smooth. Very important, the ability to avoid collisions with either static or dynamic obstacles have been demonstrated. Future work consist on implementation and validation on the physical robots.

\section{ACKOWLEDGMENTS}

This work was supported, in part, through grant POSI/SRI/38051/2001 from the Portuguese Foundation for Science and Technology (FCT), FEDER and PRODEP III. We wish to thank to W.Erlhagen, S.Monteiro, L.Louro, N.Hipólito, A.Moreira, T.Machado.

\section{References}

1. Ahamadabadi M. and Nakano E. "A cooperative multiple robot system for object lifting and transferring tasks". In Proc. ROBOMECH'96, Annual Conf. of The Japanese Society of Mechanical Eng. on Robotics and Mechatronics, 1996.

2. Aiyama Y. et al. "Cooperative transportation by two four-legged robots with implicit communication". Robotics and Autonomous Systems, 1999.

3. Bicho E., Mallet P. and Schöner G. "Target representation on an autonomous vehicle with low level sensors". The Int. Jou. of Robotics and Research, 19(5):424-447, 2000.

4. Ahmadabadi M. and Nakano E. "A constrain and move approach to distributed object manipulation". IEEE Transactions on Robotics and Automation, 17(2):157-172, 2001.

5. Chaimowicz L., Sugar T., Kumar V. and Campos V. "An architecture for tightly coupled multi-robot cooperation". in Proc. IEEE ICRA, 2292-2297, 2001.

6. Soares R., Bicho E. "Using Attractor Dynamics to Generate Decentralized Motion Control of Two Robots Transporting a Long Object in Coordination". Proc. of the 2002 IEEE/RSJ Intl. Conf. on Intelligent Robots and Systems, EPFL, Lausanne, 2002.

7. Bicho E. "Dynamic approach to Behavior-Based Robotics: design, specification, analysis, simulation and implementation". Shaker Vergal, 2000.

8. Bicho E., Louro L., et al. "Coordinated transportation with minimal explicit communication between robots". 5th IFAC Symp. on IAV, Lisbon, Portugal, July 5-7, 2004.

9. Wang Z., Takano Y., Hirata Y. and Kosuge K. "From Human to Pushing Leader Robot: Leading a Decentralized Multirobot System for Object Handling". in Proc. IEEE Int. Conf. Robotics and Biomimetics, 2004.

10. Hirata Y., Kume Y., Sawada T., Wang Z. and Kosuge K. ”Handling of an object by multiple mobile manipulators in coordination based on caster-like dynamics". in Proc. IEEE Int. Conf. Robotics and Automation, 807-812, 2004. 